\title{
Influence of active sites organisation on calcium carbonate formation at model biomolecular interfaces
}

\author{
S. Hacke ${ }^{\mathrm{a}, *}$, D. Möbius ${ }^{\mathrm{b}}$, V.-T. Lieu ${ }^{\mathrm{b}}$ \\ ${ }^{\mathrm{a}}$ Georg-August-Universität, GZG - Kristallographie, Goldschmidtstr. 1, 37077 Göttingen, Germany \\ ${ }^{\mathrm{b}}$ Max-Planck-Istitut für Biophysikalische Chemie, Am Faßberg 11, 37077 Göttingen, Germany
}

Available online 30 December 2004

\begin{abstract}
In an approach to understand the influence of structural parameters of interfaces on calcification in biomineralisation, the distribution and conformation of head groups as active sites in an inert matrix were varied using two-component phospholipid model monolayers. Dimyristoylphosphatidic acid (DMPA) and dipalmitoylphosphatidylcholin (DPPC), respectively, were the active components, and methyl octadecanoate (MOD) was used as inactive matrix. Surface pressure-area isotherms provide evidence for a different distribution of the active components in the matrix. Formation of solid calcium carbonate with twocomponent monolayers on subphases containing aqueous $\mathrm{CaCO}_{3}$ was observed in situ by Brewster angle microscopy, where $\mathrm{CaCO}_{3}$ domains appear bright. Striking differences in kinetics and extent of $\mathrm{CaCO}_{3}$ formation are observed between monolayers containing dimyristoylphosphatidic acid and those containing dipalmitoylphosphatidylcholin. The presence of $\kappa$-carrageenan in the subphase as a further active component resulted in partial inhibition of $\mathrm{CaCO}_{3}$ formation.
\end{abstract}

(C) 2004 Elsevier B.V. All rights reserved.

Keywords: Mixed model monolayers; Calcium carbonate formation; Phospholipids; Kinetics; Critical cluster

\section{Introduction}

The formation of solid calcium carbonate is an important process in biological systems at the interface between living organisms and mineral phases such as macromolecular frameworks, lipid membranes or cell walls [1-4]. A variety of synthetic (bio-) organic templates have been developed to direct

\footnotetext{
* Corresponding author. Tel.: +49 5513914624; fax: +49 551399521 .

E-mail address: shacke@gwdg.de (S. Hacke).
}

nucleation and growth of solid $\mathrm{CaCO}_{3}$ such as insoluble Langmuir monolayers of lipids with different head groups at the air-water interface $[5,6]$. The minimal lateral size of monolayer domains inducing 3-D crystal nucleation may be determined by using mixed Langmuir monolayers [7,8]. By variation of the molar ratio of the two components in such monolayers, it has been shown that domains of the active component of finite size are required to initiate crystallisation [7].

A new approach in the investigation of $\mathrm{CaCO}_{3}$ formation at interfaces is the variation of parameters 
like density and distribution of head groups as active sites in an inert matrix as well as head group conformation. In previous work, we compared different two-component model monolayers with octadecanoic acid and octadecyl succinic acid, respectively, as active components in an inert matrix of methyl octadecanoate. Striking differences in kinetics and extent of $\mathrm{CaCO}_{3}$ formation were observed in these systems in spite of the same average density of carboxyl groups $(\mathrm{COOH})$. This demonstrates the important role of the lateral distribution of active sites in the process of biomineralisation at interfaces. In particular, the observations suggest that a minimum number of active groups (a critical cluster) are required to act as nucleation site for the formation of solid $\mathrm{CaCO}_{3}$ [9].

In the present investigations of $\mathrm{CaCO}_{3}$ formation, we used a more complex molecules as active components, i.e., phospholipids due to their special applicability as models for biomembranes, and in addition, a polysaccharide as an example of a group of important polymers widely present in living organisms, to study their influence on the calcification of the model biomembrane. We have chosen the two phospholipids dimyristoylphosphatidic acid (DMPA) with the phosphate head group as active site and dipalmitoylphosphatidylcholin (DPPC) with zwitterionic head group as active sites and methyl octadecanoate (MOD) as inactive matrix for the variation of the average surface density of the active groups.

Brewster angle microscopy (BAM) has been used for studying the calcium carbonate precipitation with monolayers of long chain fatty acids of various chain lengths [10], and in situ $\mathrm{CaCO}_{3}$ formation as a function of active sites distribution of two-component lipid model monolayers $[9,11]$.

\section{Experimental}

\subsection{Materials}

Dimyristoylphosphatidic acid and dipalmitoylphosphatidylcholin were purchased from Sigma Chemicals Co., U.S.A., methyl octadecanoate from E. Merck, Germany. All lipids were used without further purification and dissolved in chloroform (Baker
Chemicals, HPLC grade) to form 1-mM spreading solutions. Water (resistance $18 \mathrm{M} \Omega \mathrm{cm}$ ) purified in a Milli-Q system (Millipore Composition) was used for preparing the aqueous $4 \mathrm{mM} \mathrm{CaCO}_{3}$ solutions as the subphase. Calcium chloride tetrahydrate, sodium hydrogen carbonate and sodium chloride (all suprapure quality) were obtained from E. Merck, Germany. The natural polysaccharide $\kappa$-carrageenan, isolated from red algea (Rhodophyta) $\left(M_{\mathrm{w}}=441.342 \mathrm{~g} / \mathrm{mol}\right.$ per $(\mathrm{AB})_{n}$ repeat unit), was purchased from Sigma Chemicals Co., U.S.A. The aqueous $4 \mathrm{mM} \mathrm{CaCO}_{3}$ (without or with $0.4 \mathrm{mM}$ k-carrageenan) solutions were prepared by mixing equal volumina of $\mathrm{NaHCO}_{3}$ (without or with $0.8 \mathrm{mM}$ K-carrageenan) and $\mathrm{CaCl}_{2} \cdot 4 \mathrm{H}_{2} \mathrm{O}$ solutions $(8 \mathrm{mM}$ in $0.1 \mathrm{M} \mathrm{NaCl})$ at $20{ }^{\circ} \mathrm{C}$.
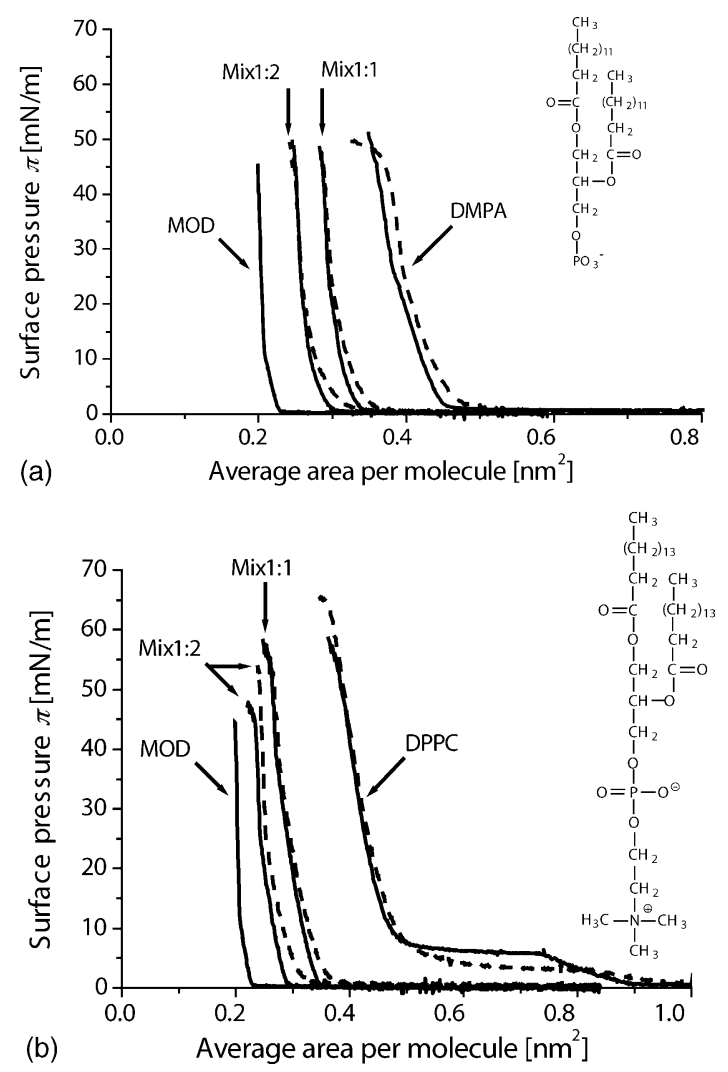

Fig. 1. Characterisation of monolayers at the air-solution interface by surface pressure $(\pi)$-area $(A)$ isotherms. (a) Model Monolayer DMPA/MOD, (b) model monolayer DPPC/MOD. The pure monolayers and molar mixing ratios indicated at the curves. Subphase: $4 \mathrm{mM} \mathrm{CaCO}_{3}$ (full lines) and with additional $0.4 \mathrm{mM} \mathrm{\kappa}$-carrageenan (dashed lines); $20{ }^{\circ} \mathrm{C}$. 


\subsection{Procedures}

The $\mathrm{CaCO}_{3}$ solutions were poured into a custom built PTFE trough $(74 \mathrm{~cm} \times 10 \mathrm{~cm})$ with a maximum working area of $590 \mathrm{~cm}^{2}$ and two simultaneously moving barriers.

Monolayers were formed by spreading $90 \mu \mathrm{l}$ of solutions of lipids or mixtures of these, respectively (except pure MOD with $180 \mu \mathrm{l}$ ), in chloroform on subphases containing $\mathrm{CaCO}_{3}$ solutions ( \pm k-carrageenan) at $20{ }^{\circ} \mathrm{C}$ with a microsyring. The surface pressure $\pi$ was measured with a filter paper (Schleicher \& Schüll 589) Wilhelmy balance and was kept constant after reaching $\pi=25 \mathrm{mN} \mathrm{m}^{-1}$ by a feedback loop. The speed of monolayer compression was $20.7 \mathrm{~cm}^{2} \mathrm{~min}^{-1}$. The formation of solid $\mathrm{CaCO}_{3}$ in the head group region of monolayers at the air-solution interface was followed in situ using a modified Imaging Ellipsometer I-Elli 2000 from Nanofilm Technologie, Göttingen, with a $532 \mathrm{~nm}$ laser diode (20 mW, Uniphase).
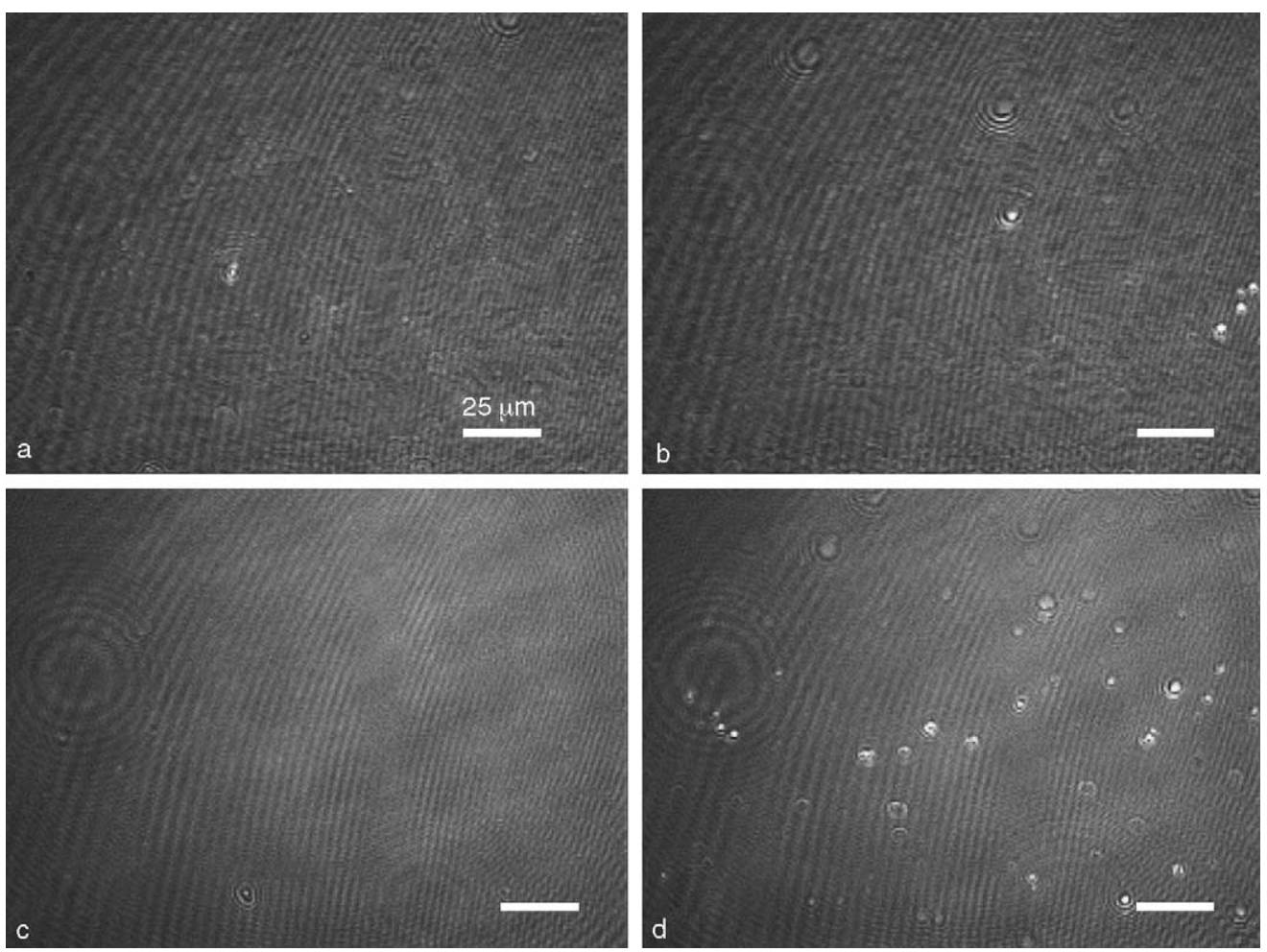

Fig. 2. Observation of $\mathrm{CaCO}_{3}$ formation by Brewster angle microscopy. BAM images of model monolayers on top of $\mathrm{CaCO}_{3}$ solutions taken at different times $t$ (no additional $\kappa$-carrageenan). DMPA:MOD $=1: 2$ (a) $t=20 \mathrm{~min}$, (b) $t=45 \mathrm{~min}$; DPPC:MOD $=1: 2$ (c) $t=20 \mathrm{~min},(\mathrm{~d})$ $t=45 \mathrm{~min}$; images taken at constant monolayer area $\left(\pi=25 \mathrm{~m} \mathrm{~N} \mathrm{~m}^{-1}\right) ; 20{ }^{\circ} \mathrm{C}$. 
of pure DPPC shows the well-known phase transition in the range $0.5 \mathrm{~nm}^{2} \leq A \leq 0.75 \mathrm{~nm}^{2}$ with very little increase of surface pressure upon compression (Fig. 1b). In contrast to the behaviour of DMPA mixtures at $\pi=25 \mathrm{mN} \mathrm{m}^{-1}$ the observed average area per molecule clearly deviates in the case of DPPC from ideal behaviour (plots not shown). In presence of $\kappa$-carrageenan in the subphase the area per amphiphile is slightly increased for both phospholipid model systems presumably due to the interaction of the polysaccharide with the monolayer as well as competitive interactions of the monolayer head groups and the anionic groups of the polysaccharide with the calcium ions in the subphase.

The Brewster angle microscope images of the twocomponent monolayers shown in Fig. 2 were taken at different times $t$ after starting monolayer compression (the first image recorded when the surface pressure $\pi$ has reached $25 \mathrm{mN} \mathrm{m}^{-1}$, i.e., after $20 \mathrm{~min}$, and the second image after $45 \mathrm{~min}$ ). They clearly show the formation and growth of bright domains (Fig. 2). The images at $\pi=25 \mathrm{mN} \mathrm{m}^{-1}$ of the monolayers DMPA:$\mathrm{MOD}=1: 2$ (Fig. 2a and b) and DPPC:MOD $=1: 2$ (Fig. 2c and d), reveal striking differences between the model systems. Many more bright domains indicating the formation of an ultrathin layer of $\mathrm{CaCO}_{3}$ in contact with the monolayer head groups are observed for DMPA:MOD $=1: 2$ (Fig. 2a, $t=20 \mathrm{~min}$ ) than for DPPC:MOD $=1: 2$ (Fig. 2c, $t=20 \mathrm{~min}$ ). Further, the time evolution differs dramatically for the two monolayer compared here. The area fraction covered with bright domains at $t=45$ min obviously is much larger for the monolayer with DPPC (Fig. 2d) than with DMPA (Fig. 2b). The topography of the monolayers DMPA:MOD =1:2 seen in the BAM images may indicate a phase separation of the two components (i.e. DMPA and MOD) in this particular model system.

We have adapted to our systems a growth model used in the determination of heterogeneous nucleation rates [12]. A Gaussian size distribution of the bright domains of $\mathrm{CaCO}_{3}$ in contact with the monolayer head groups is assumed. As the distribution shifts with time in size and thickness of the ultrathin layer, the domains become visible in the BAM exceeding a fixed brightness level. The time dependence of the average area fraction of bright domains in the monolayer then is the integral of the Gaussian distribution [9], i.e., the
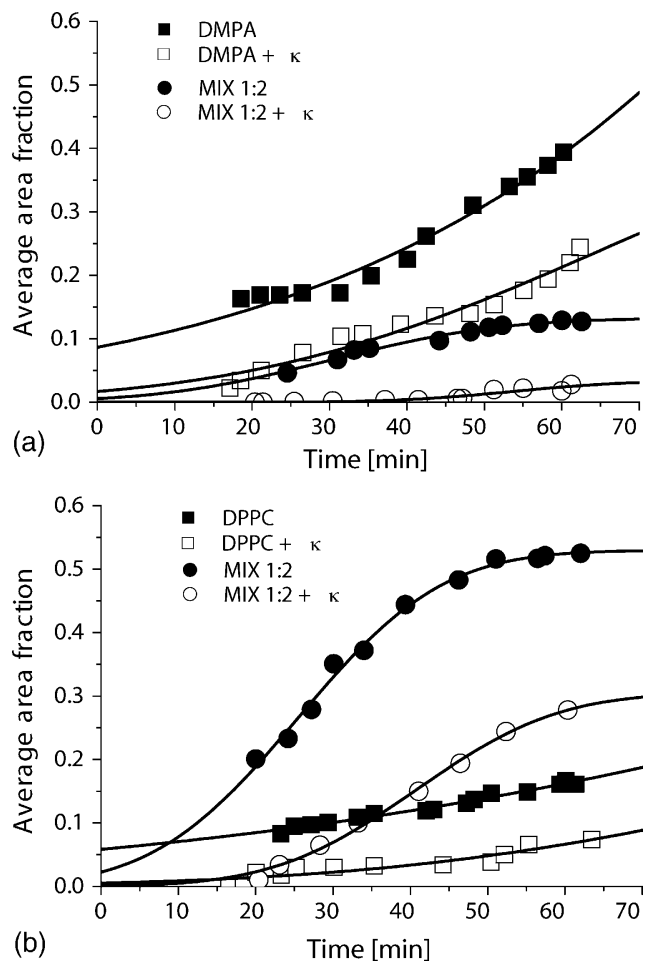

Fig. 3. Kinetics of $\mathrm{CaCO}_{3}$ formation at two-component monolayers. Average area fraction of bright domains determined from BAM images as function of time $t$ for the monolayers: (a) pure DMPA and DMPA:MOD $=1: 2$ and (b) pure DPPC and DPPC:MOD $=1: 2$, respectively, as indicated; the full curves are least squares fits; images taken at constant monolayer area $\left(\pi=25 \mathrm{mN} \mathrm{m}^{-1}\right)$. Subphase: $4 \mathrm{mM} \mathrm{CaCO}_{3}$ (filled symbols) and with additional $0.4 \mathrm{mM} \kappa$-carrageenan (open symbols); $20{ }^{\circ} \mathrm{C}$.

error function. The different series of images taken with increasing time have been evaluated by determining the average area fraction of domains exceeding a limiting brightness value see Fig. 3 . The results clearly indicate the fundamentally different behaviour of the model system DMPA:MOD $=1: 2$ (Fig. 3a) and DPPC:MOD = 1:2 (Fig. 3b), respectively, concerning kinetics of formation and area fraction of the bright domains extrapolated to very long times (Fig. 3, the error functions fitted to the experimental points are is illustrated as solid lines). The area fraction of the bright domains is reduced as expected in the model system DMPA:MOD $=1: 2$ as compared to pure DMPA. In contrast, an enhancement of this fraction is observed for the model system DPPC:MOD $=1: 2$ as compared to pure DPPC. This 
surprising phenomenon may be attributed to the differences in accessibility of the phosphate group considered as the nucleation site to the $\mathrm{Ca}^{2+}$ ions. One reason might be the different organisation of the head groups, due to an additional cholin-group of DPPC with respect to DMPA. In the case of a pure DPPC monolayer in the condensed state, the cholin group screens the phosphate. In case of molecular mixing of the components of the DPPC:MOD $=1: 2$ monolayer (Fig. 1b), the inactive component (MOD) acts as spacer that reduces the screening of the phosphate group by the cholin and provides improved conditions for interaction of the phosphate group with the $\mathrm{Ca}^{2+}$ ions in the subphase. The addition of $\kappa$-carrageenan in the subphase reduces crystallisation for both DMPA and DPPC systems, presumably due to competition with the phosphate group.

\section{Conclusion}

The formation of $\mathrm{CaCO}_{3}$ in the head group region of insoluble two-component model monolayers at the solution-air interface has been observed in situ by Brewster angle microscopy. The two phospholipids DMPA and DPPC with different head groups have been compared as pure monolayers as well as in mixtures with methyl octadecanoate. DPPC is miscible with MOD, whereas a phase separation of DMPA and MOD occurs. Striking differences in kinetics and extent of $\mathrm{CaCO}_{3}$ formation observed in the twocomponent systems, due to improved accessibility of the phosphate group of DPPC as compared to pure
DPPC monolayer. With DMPA a reduction of crystallisation is observed in the two-component monolayer as compared to pure DMPA. The addition of the polysaccharide $\mathrm{\kappa}$-carrageenan to the aqueous subphase causes a reduction of crystallisation due to competition between the sulfate groups of the polysaccharide and the phosphate groups of the insoluble monolayers.

\section{References}

[1] S. Weiner, W. Traub, H.A. Lowenstam, Organic matrix in calcified exoskeletons, in: P. Westbroek, E.W. de Jong (Eds.), Biomineralization and Biological Metal Accumulation, D. Reidel Publisher Comp., Dordrecht, 1983, pp. 205-224.

[2] H.A. Lowenstam, S. Weiner, On Biomineralization, Oxford University Press, New York, 1989.

[3] K. Simkiss, K. Wilbur, Biomineralization: Cell biology and Mineral Deposition, Academic Press, San Diego, 1989.

[4] S. Weiner, L. Addadi, H.D. Wagner, Mater. Sci. Eng. C 11 (2000) 1-8.

[5] B.R. Heywood, S. Mann, Adv. Mater. 6 (1994) 9-20.

[6] A.L. Litvin, S. Valiyaveettil, D.L. Kaplan, S. Mann, Adv. Mater. 9 (1997) 124-127.

[7] I. Weissbuch, J. Majewski, K. Kjaer, J. Als-Nielsen, M. Lahav, L. Leiserowitz, J. Phys. Chem. 97 (1993) 12848-12857.

[8] I. Kuzmenko, H. Rapaport, K. Kjaer, J. Als-Nielsen, I. Weissbuch, M. Lahav, L. Leiserowitz, Chem. Rev. 101 (2001) 16591696.

[9] S. Hacke, D. Möbius, Colloid \& Polymer Science (2004) No 10.1007/s00396-004-1074-7.

[10] E. Loste, E. Diaz-Marti, A. Zarbakhsh, F.C. Meldrum, Langmuir 19 (2003) 2830-2837.

[11] S. Hacke, Ph.D. Thesis, University Göttingen, Germany, 2001.

[12] H. Schubert, A. Mersmann, Trans. I Chem. E 74 A (1996) 821827. 